\title{
Relationship between aquaporins expression and B concentration for conferring cold stress tolerance in broccoli cultivars
}

\author{
Alvaro Lopez-Zaplana, Juan Nicolas-Espinosa, Micaela Carvajal, Gloria Bárzana * \\ Aquaporins Group, Plant Nutrition Department, Centro de Edafología y Biología Aplicada del Segura (CEBAS-CSIC), Campus Universitario de Espinardo, Edificio 25, \\ 30100, Murcia, Spain
}

\section{A R T I C L E I N F O}

\section{Keywords:}

Broccoli

Cold tolerance

Aquaporins

Water relations

Boron

Physiological response

\begin{abstract}
A B S T R A C T
Broccoli is an important crop worldwide with numerous nutritional benefits. Three cultivars (Naxos, Triton and Mykonos) grown in the winter in Spain and highly throughout Europe has been investigated, since they have to deal with the great variation in temperature between day and night and often suffers from night frosts. As, there is no physiological or molecular studies that allows to elucidate the mechanisms involved in its ability to resist cold, in this study, we seek to determine the mineral content, water relations and gas exchange response, together with aquaporins expression in the three different cultivars of broccoli against a recurring temperature of $4{ }^{\circ} \mathrm{C}$ at night. A clear cause-effect relationship is shown regarding the control of aquaporins and the ability to deal with cold stress, as well as the importance of boron in this process in broccoli. Specifically, the expression of aquaporins in each cultivar showed different patterns according to the three different strategies utilized to cope with cold stress, but the cultivars adapted to the cold showed high levels of aquaporins expression after a low temperature treatment. Furthermore, aquaporin NIP6 seems to be decisive for maintaining adequate boron levels in the leaves in accordance to resistance to cold conditions.
\end{abstract}

\section{Introduction}

In the last few years, Brassica oleracea L. var. Italica (broccoli) has experienced a very important increase in their world production, from 16 million to 26 million tons from the year 2000-2017. In 2017, Spain ranked fourth in total global world production per ton (Food and agriculture, Organization of the United Nations, http://www.fao.org/ faostat). The economic interest of broccoli increased every year around the world (Francisco et al., 2017), mainly due to its numerous beneficial properties due to its high concentrations of vitamins A, C, E, and $\mathrm{K}$, minerals, and important secondary metabolites such as glucosinolates and phenolic compounds (Moreno et al., 2006). Cultivars of Broccoli have been agronomically selected to cover a longer production period and are therefore commonly grown throughout the year in the Mediterranean region. However, frost and low temperatures often reduces its growth and yield during winter (Jeon and Kim, 2013; Nasehzadeh and Ellis, 2017) especially affecting the cultivars grown in this cold season.

Exposure to cold temperatures produces numerous changes in plants, ranging from decreased water intake and transpiration rate, altered water relations and potentials, and increased osmotic pressure through the production of osmolytes to prevent cell damage (Sanghera et al., 2011; Wong et al., 2006). These changes not only occur at the physiological level, but gene expression is also affected, in genes related to stress response, hormonal metabolism, nutrient transporters, and aquaporins for water and nutrient transport (Fowler and Thomashow, 2002; Jang et al., 2004).

In general, plants have a great need for water regulation for adapting to environmental fluctuations (Osakabe et al., 2014). The absorption and establishment of a continuous flow of water from the soil to the entire plant, and the subsequent evaporation in the atmosphere is crucial for the physiological activities of plants and for handling the stress imposed by the environment, resulting in a continuous soil-plant-atmosphere system (Steudle, 2001). The transport of water through the entire plant system could be performed through two different pathways: the apoplastic path (via the hydrostatic gradient generated by transpiration), and the cell-to-cell path (generated by an osmotic gradient). The latter is where we find the importance of aquaporins, as their action allows the flow of water through lignin and suberin barriers and cell membranes (Maurel et al., 2008).

Aquaporins are transmembrane proteins that mainly mediate the transport of water, but other small solutes such as glycerol, urea, carbon

\footnotetext{
* Corresponding author.

E-mail address: gbarzana@cebas.csic.es (G. Bárzana).
} 
dioxide, ammonia $\left(\mathrm{NH}_{3}\right)$, metalloids, and reactive oxygen species (ROS) can also be transported by some of them (Maurel et al., 2015). Aquaporins are phylogenetically classified in higher plants into five subgroups: plasma membrane intrinsic proteins (PIPs), tonoplast intrinsic proteins (TIPs), nodulin-26-like intrinsic proteins (NIPs), small basic intrinsic proteins (SIPs), and the uncharacterized subfamily, $\mathrm{X}$ intrinsic proteins (XIPs) (Barzana et al., 2020).

As cold conditions are known to affect hydraulic conductivity in roots and cells in the entire plant, aquaporins have become important in the study of cold as an abiotic stress (Aroca et al., 2005), and previous studies have shown that the regulation of aquaporins is a defence mechanism against the cold (Ahamed et al., 2012; Huang et al., 2014). Thus, it is well known that cold stress conditions diminish the expression of some aquaporins (Jang et al., 2004; Murai-Hatano et al., 2008), thereby affecting water regulation, water relations and subsequently, plant growth (Ding et al., 2020). Nevertheless, the reduction of aquaporins expression not only affects water transport but also the transport of other important nutrients such as nitrogen $(\mathrm{N})$, silicon $(\mathrm{Si})$ or boron (B), as they are also transported by aquaporins (Bienert et al., 2008). Among these nutrients, boron is becoming of great interest in the response of plants to cold.

$B$ is an essential element for plants, and is associated with the structure and well-functioning of the plant cell wall due to its capacity to form borate esters with apiose residues of rhamnogalacturonan II (RG-II) (Camacho-Cristóbal et al., 2008). Cold conditions can induce changes in cell membranes, altering their composition and rigidity (Miura and Furumoto, 2013). B has been shown to increase the resistance of some crops against the cold by reducing some deleterious effects of cold stress (Dezhabad and Haghighi, 2020; Huang et al., 2005; Köse et al., 2018), and a recent study showed the relation of cold stress, the aquaporin BvCOLD1, and B (Porcel et al., 2018), but the manners in which boron interacts, or its involvement in the existence of varieties that are more resistant than others, are unknown. In general, some NIPs are indispensable for B uptake and transport in the form of boric acid, such as AtNIP5;1 and AtNIP6;1 (Takano et al., 2006; Tanaka et al., 2008; Wang et al., 2016). On the other hand, some PIPs, such as OsPIP1;3, OsPIP2;4, OsPIP2;6, and OsPIP2;7 are also involved in both influx and efflux of boric acid through the plasma membrane (Kumar et al., 2014; Mosa et al., 2016). Finally, for the adequate transport of B, a continuous flow of water generated by the rest of the aquaporins is necessary (Aroca et al., 2001; Miura and Furumoto, 2013; Tyerman et al., 2002).

For these reasons, the aim of this work was to study the relationships between the different physiological responses to night-time cold stress and their subsequent recovery in three different broccoli cultivars (Naxos, Triton, and Mykonos), as well as the molecular mechanisms involved in its different ability to deal with cold stress. Thus, based on the importance of aquaporins as mediators of the stress response, we measured the expression profiles of PIP1, PIP2, TIP1, and TIP2 to analyse the transport of water, and the expression profiles of NIP5 and NIP6 related to their possible involvement in B transport and concentration in different parts of the plants.

\section{Material and methods}

\subsection{Plant material and growth conditions}

Seeds of broccoli plants (Brassica oleracea L. var. Italica) from the three cultivars named Naxos, Triton and Mykonos (SAKATA) were prehydrated with deionised water and aerated continuously for $12 \mathrm{~h}$. After this, the seeds were germinated in vermiculite, in the dark at $28^{\circ} \mathrm{C}$, for 2 $\mathrm{d}$. They were then transferred to a controlled-environment chamber and placed in $15 \mathrm{~L}$ containers with continuously-aerated Hoagland's nutrient solution (Bussler, 2007), which was renewed every week. The chamber had a 16-h light and 8-h dark cycle with temperatures of 25 and $20{ }^{\circ} \mathrm{C}$ and relative humidity of $60 \%$ and $80 \%$, respectively. A photosynthetically-active radiation (PAR) of $400 \mu \mathrm{mol} \mathrm{m}^{-2} \mathrm{~s}^{-1}$ was provided by LEDs.

After 2 weeks of growth, 48 plants ( 16 of each broccoli cultivar) were grouped into subgroups of $n=8$ each one. One subgroup of each cultivar was used as a control and was kept under these control conditions for the remaining 2 weeks of the experiment, and the other subgroup (from now on low temperature treatment (LT)), was placed in a cold room at $4{ }^{\circ} \mathrm{C}$ every night $(14 \mathrm{~h})$ until the end of the experiment. All the measurements were made the following day, $2 \mathrm{~h}$ after cold treatment. The experimental design was completed randomized design (CRD). The three cultivars were distributed in six different containers randomly placed into the chamber.

\subsection{Relative water content (RWC)}

The relative water content was calculated from the fresh weight, fullturgor weight, and dry weight of a $1 \mathrm{~cm}^{2}$ fragment from 3 to 4 fully developed leaves. For the turgor weight, the fragments were kept in darkness and humidity for $12 \mathrm{~h}$. For the dry weight, the fragments were placed in an oven for 3 days, until they kept their weight unchanged.

\subsection{Transpiration rate}

Transpiration rate $\left(\mathrm{mmol} \mathrm{m} \mathrm{m}^{-2} \mathrm{~s}^{-1}\right)$ was measured in the second, third, and fourth fully expanded leaves using the TPS-2 Portable Photosynthesis System (PP Systems, Inc., MA, Amesbury, USA).

\subsection{Leaf water potential $\left(\Psi_{\omega}\right)$ and osmotic potential $\left(\Psi_{\pi}\right)$}

The leaf water potential $\left(\Psi_{\omega}\right)$ of the fourth fully-expanded leaf was measured using the pressure chamber technique (Turner, 1988). The osmotic potential $(\Psi \pi)$ of leaves and roots was measured using an osmometer (Digital Osmometer, Roebling, Berlin) at $25 \pm 1{ }^{\circ} \mathrm{C}$.

\subsection{Root hydraulic conductivity $\left(L_{0}\right)$}

Root hydraulic conductivity $\left(\mathrm{L}_{0}\right)$ was measured on detached roots exuding under atmospheric pressure for $15 \mathrm{~min}$ (Aroca et al., 2007). $\mathrm{L}_{o}$ was calculated as $\mathrm{L}_{0}=\mathrm{J}_{\mathrm{V}} / \Delta \Psi$, were $\mathrm{J}_{\mathrm{v}}$ is the exuded sap flow rate and $\Delta \Psi$ the osmotic potential difference between the exuded sap and the nutrient solution into which the plants were placed. The measurements were carried out $4 \mathrm{~h}$ after the onset of light. The $\mathrm{L}_{0}$ value was expressed in $\mathrm{mg} \mathrm{H}_{2} \mathrm{O} \mathrm{g} \mathrm{RDW}{ }^{-1} \mathrm{MPa}^{-1} \mathrm{~h}^{-1}$.

\subsection{RNA extraction and $c D N A$ synthesis}

Total RNA was extracted using the RNeasy Plant Mini Kit (Qiagen, Hilden, Germany), according to the manufacturer's protocol. The concentration and quality of RNA were checked using a NanoDrop 1000 Spectrophotometer (Thermo Scientific). Contaminating DNA was removed with DNase I using a DNA-free Kit (Ambion, Applied Biosystems, Austin, TX, USA), and the RNA concentration was quantified with a Nanodrop 1000 Spectrophotometer (Thermo Scientific). The RNA extracted was stored at $-80^{\circ} \mathrm{C}$ until used. The M-MLV reverse transcriptase from the RETROscript Kit (Ambion, Applied Biosystems) was used to synthesise cDNA from $2 \mu \mathrm{g}$ of total RNA. cDNA synthesis was carried out with heat denaturation of the RNA, according to the manufacturer's instructions.

\subsection{Primer design}

Primers for PIP1 and PIP2 subgroups were kindly provided by Muries et al. (2011). As the genome of Brassica oleracea var. Italica is not yet known, a general search in the NCBI database of all the aquaporin sequences available for the Brassica oleracea family was obtained and grouped into general aquaporin subgroups. Sequences from Brassica oleracea var. oleracea (Diehn et al., 2015) were also included. Then, 
primers were designed based on the alignment of all the sequences included in each of the subgroups (TIP1, TIP2, NIP5, and NIP6), obtaining a maximum intra-varieties homology ranging from 90 to $100 \%$ with all the members of the Brassica oleracea aquaporin genes in the subgroup, and discarding the results with more than $75 \%$ homology with any other aquaporin gene from all the other aquaporin subgroups. The primer design was carried out manually, meeting the specific requirements imposed by the high homology of the sequences used, and the technique used for the analysis. A virtual analysis of melting temperature, primer hairpins, self-dimer, hetero-dimer, individual and total $\Delta \mathrm{G}$ was conducted with the PCR Primer Stats software (https://www. bioinformatics.org/sms2/pcr_primer_st), and IDT Oligo Analyzer Tools (https://eu.idtdna.com/calc/analyzer/). The $\Delta \mathrm{G}$ accepted for dimmer analysis was always less than $-7 \mathrm{kcal} / \mathrm{mole}$.

The qPCR analysis of each primer pair revealed perfect amplification of a single isoform for each subgroup. However, we cannot identify the specific gene within each group. Therefore, we will refer to "group gene", hereinafter: PIP1, PIP2, TIP1, TIP2, NIP5 and NIP6. The efficiency of the primer sets was evaluated with the QuantStudio 5 software (QuantStudio TM Design and Analysis Software version 1.4.0.0) by analysing the ratio cycle threshold $(\mathrm{Ct}) /$ fluorescence at six independent points in the PCR curves (Ramakers et al., 2003), obtaining values between 97 and $100 \%$ (Table 1 ).

Five housekeeping primers, $18 \mathrm{~s}$ Ribosomal RNA for B. oleracea var. Italica (Muries et al., 2011), Tubulin beta6 (TUB6; gi: KF218597), SAND family protein (SAND1; gi: KF218596), Ubiquitin2 (UBQ2; gi: KF218593), and TATA-box-binding protein 1 (TBP1; gi: KF218595) for B. oleracea, were selected according to Brulle et al. (2014), checked in every cDNA used for quantitative PCR quantification (qPCR), and measured using a Visual Basic application for Excel (GeNorm), which automatically calculates the gene stability (Vandesompele et al., 2002). From all of them, the only one reliable in our samples was $18 \mathrm{~s}$ Ribosomal RNA, which was also presented as the most stable gene according to the analysis carried out with the Visual Basic application for Excel (GeNorm). For these two reasons, the $18 \mathrm{~s}$ Ribosomal RNA was selected as the reference gene for the standardisation of each sample.

\subsection{Quantitative real-time PCR (QRT-PCR) analyses}

To compare the general expression of aquaporins in the different cultivars and treatments, RT-qPCR was carried out. It was performed using $2 \mu \mathrm{L}$ of $1: 10$ diluted cDNA samples in $8 \mu \mathrm{L}$ of reaction medium containing $600 \mathrm{nM}$ gene-group primers (Table 1), and $5 \mu \mathrm{L}$ of SYBR Green Master Mix 2X (Applied Biosystems), in an Applied Biosystems

Table 1

Primers used for measurement of $B$. oleracea var. italica aquaporins expression by PCR and qRT-PCR. Columns: gene name, primer sequence: top: forward primer (F), and bottom, reverse (R) primer, and \% efficiency.

\begin{tabular}{|c|c|c|}
\hline Genes name & $\begin{array}{l}\text { Primer sequence (Forward/Reverse } \\
\text { primer) }\end{array}$ & $\begin{array}{l}\text { Efficiency } \\
(\%)\end{array}$ \\
\hline PIP1* & $\begin{array}{l}\text { 5'-GCGGTATCGCCGAGTTCA-3' } \\
\text { 5'-GGTCAACACGGTGATGTAAGGA-3' }\end{array}$ & 90 \\
\hline PIP2* & $\begin{array}{l}\text { 5'-CCTCGCCGCAGAGATCA-3' } \\
\text { 5'-TGGCGGAGAAGACGGTGTAG-3' }\end{array}$ & 90 \\
\hline TIP1 & $\begin{array}{l}\text { 5'-TGGTATCCTCTACTGGATTGC-3' } \\
\text { 5'-CAAAGGTCATCACGATCTCG-3' }\end{array}$ & 100 \\
\hline TIP2 & $\begin{array}{l}\text { 5'-GAAGGAGTAGTGATGGAGAT-3' } \\
\text { 5'-GAACCTTTCTTGGGATCAGC-3' }\end{array}$ & 100 \\
\hline NIP5 & $\begin{array}{l}\text { 5'-GAACATTCATCTTGATATTCAC-3' } \\
\text { 5'-TAGAGCTGCGAATGCTATGG-3' }\end{array}$ & 97.7 \\
\hline NIP6 & $\begin{array}{l}\text { 5'-AAAGAGGAATGGACATAATGG-3' } \\
\text { 5'-CGTTAGGAGGAGGTAGAGC-3' }\end{array}$ & 99.4 \\
\hline 18 s ribosomal RNA & $\begin{array}{l}\text { 5'-CCTGCGGCTTAATTTGACTC-3' } \\
\text { 5'-AGACAAATCGCTCCACCAAC-3' }\end{array}$ & 90 \\
\hline
\end{tabular}

* PIP1, PIP2 and 18 ribosomal RNA primers obtained from Muries et al. (2011).
7500 Real-Time PCR system. After denaturation at $95^{\circ} \mathrm{C}$ for $10 \mathrm{~min}$, amplification occurred in a two-step procedure: $15 \mathrm{~s}$ of denaturation at $95^{\circ} \mathrm{C}$ and $1 \mathrm{~min}$ of annealing and extension at $60^{\circ} \mathrm{C}$ for 40 cycles, followed by a dissociation stage. Data collection was carried out at the end of each round in step 2 . These conditions were used for both target and reference genes. The amplifications were performed on three independent samples for each treatment (biological replicates) and triplicate reactions were carried out for each sample (technical replicates) in 96well plates. Transcript levels were calculated using the $2^{-\Delta \Delta \mathrm{Ct}}$ method (Livak and Schmittgen, 2001), for both target and reference genes.

\subsection{Analysis of mineral elements}

Concentrations of macronutrients and micronutrients were calculated in oven-dried plant material samples, distinguishing between roots and shoots, which had been ground with a mill grinder IKA model A10 (Staufen, Germany) to obtain particle sizes between $0.5-0.7 \mathrm{~mm}$. The samples were digested in a microwave oven (CEM Mars Xpress, Mattheus, NC, USA) with a $\mathrm{HNO}_{3}-\mathrm{HClO}_{4}(2: 1)$ acid digestion. The elemental analysis was carried out using a Perkin-Elmer (Waltham, MA) 5500 model ICP emission spectrophotometer (Iris Intrepid II, Thermo Electron Corporation, Franklin, USA), at $589 \mathrm{~nm}$. The concentration was expressed as $\mathrm{mg} \mathrm{kg}^{-1} \mathrm{DW}$.

\subsection{Data analysis}

The statistical analysis of the expression of the aquaporins was carried out with 72 variables (4 plants $\mathrm{x} 2$ conditions $\times 3$ varieties $\times 3$ analytical replicates). The analysis of the rest of physiological parameters was carried out with 30 variables ( 5 plants $\times 2$ conditions $\times 3$ varieties). Statistical analyses were performed using the SPSS 25.0.0.1 software package. All the data was subjected to a Kolmogorov-Smirnov normality test. All the parameters were analysed using a one-way ANOVA, followed by Duncan's multiple comparison test. Significant differences between the values of all the parameters were determined at $\mathrm{p} \leq 0.05$, according to Duncan's test. The values presented are the means \pm standard error of the mean (SEM).

\section{Results}

\subsection{Fresh and dry weight and water content}

The values of fresh and dry weight under control conditions were higher in the Mykonos cultivar, followed by Naxos and lastly, Triton. Both the fresh weight and the dry weight showed similar responses to the low temperature treatment, as indicated by reductions under LT conditions in the three cultivars (Fig. 1). However, while the decrease was significant for the dry weight in all cultivars, the fresh weight reduction was only significant for Naxos and Mikonos, but not for Triton. Regarding the relative water content (Fig. 1), Mykonos was the only cultivar that showed a reduction after the low temperature treatment with respect to its control.

\subsection{Physiological parameters}

The transpiration rate (Fig. 2a) was significantly different between Naxos and Triton in the control treatment, being greater in the latter. With the low temperature treatment (LT), only Mykonos and Triton cultivars showed a significant decrease in this parameter as compared to the control, while Naxos plants maintained the transpiration rate at similar levels as the control treatment.

As for the water potential in leaves (Fig. 2b) Naxos control plants obtained the highest levels followed by Triton, with Mykonos showing the lowest values. The difference between Mykonos and Naxos was shown to be significant. The water potential of the 3 varieties in the LT treatments was reduced, although it was only significant in the Triton 


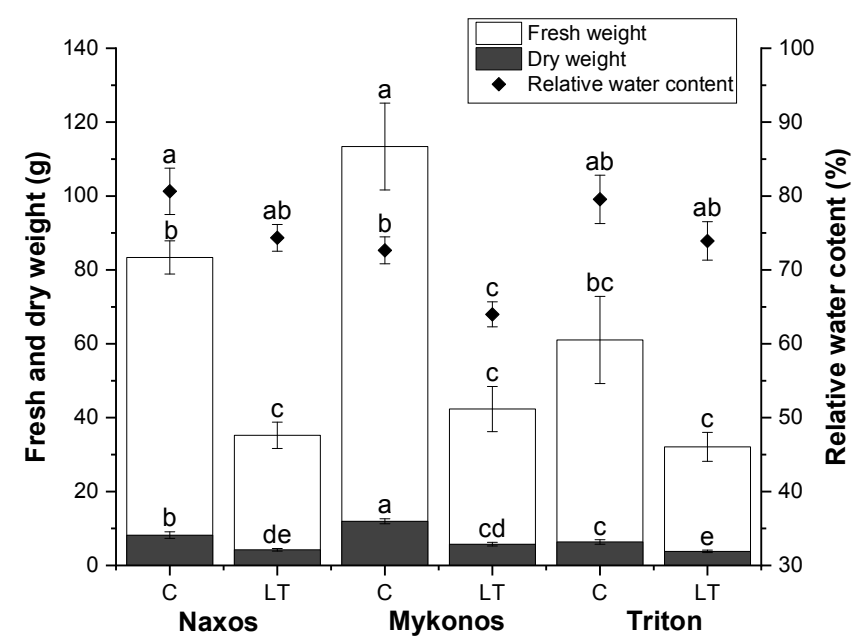

Fig. 1. Fresh weight, dry weight and relative water content (RWC) in control conditions (C) and low temperature conditions (LT) for three varieties (Naxos, Mykonos and Triton). Each bar represents the mean of 5 biological replicates \pm SEM. Columns with different letters differ significantly according to Duncan's test $(P=0.05)$.

cultivar.

The leaf osmotic potential (Fig. 2c) was found to the highest under control conditions in the Mykonos cultivar. There were no significant differences between varieties. Under the LT treatment, only a significant increase in the Triton plants with respect to its control was observed.

Regarding osmotic potential in the roots (Fig. 2d), Triton obtained the lowest values under control conditions, and these were significantly lower than the other two varieties. The root osmotic potential was significantly reduced in the Mykonos cultivar with respect to its control, in Triton it increased with the LT treatment, while no changes were observed for Naxos.
The root hydraulic conductivity $\left(\mathrm{L}_{\mathrm{o}}\right.$ ) (Fig. 2e) under control conditions was slightly higher in Mykonos, with non-significant differences between varieties. $\mathrm{L}_{0}$ increased in the Triton cultivar under LT conditions, while Mykonos and Naxos did not have significant changes in LTtreated plants compared to the controls.

\subsection{Analysis of boron}

The concentration of B was measured in leaves (Fig. 3a) and roots (Fig. 3b). In the three cultivars, the leaf concentrations were higher than in roots. In leaves (Fig. 3a), Naxos had the highest significant levels in control conditions. Furthermore, Naxos had a significant reduction in B concentration in the LT treatment with respect to its control, whilst Mykonos and Triton had similar levels. In roots (Fig. 3b) under control conditions, there were not significant differences between varieties. In this case, Naxos and Mykonos showed a significant reduction in B concentration in the LT treatment, while Triton had no significant differences.

\subsection{Aquaporins expression}

The relative expression (RE) of the aquaporins (Figs. 4 and 5) was measured. The highest constitutive expression values in leaves under control conditions for PIP1 and PIP2, were found in Mykonos (Fig. 4a, b); for TIP1, TIP2, NIP5, and NIP6 in Triton cultivars (Fig. 4c, d, e, f), and in roots, PIP1, PIP2, TIP1, TIP2, and NIP5 in Triton (Fig. 5a, b, c, d, e) with clear differences with the other two varieties. NIP6 obtained high similar expression values in Naxos and Triton roots (Fig. 5f).

In leaves (Fig. 4), a significant increase was observed in the Naxos cultivar for both PIP1 (Fig. 4a) and PIP2 (Fig. 4b) under the LT treatment as compared with the control. For the rest of the varieties, nonsignificant differences were found between treatments in these aquaporin subgroups. No significant differences were found for TIP1 (Fig. 4c) between C and LT. For TIP2 (Fig. 4d), with the LT treatment, there was a significant increase in expression in Naxos, without differences between
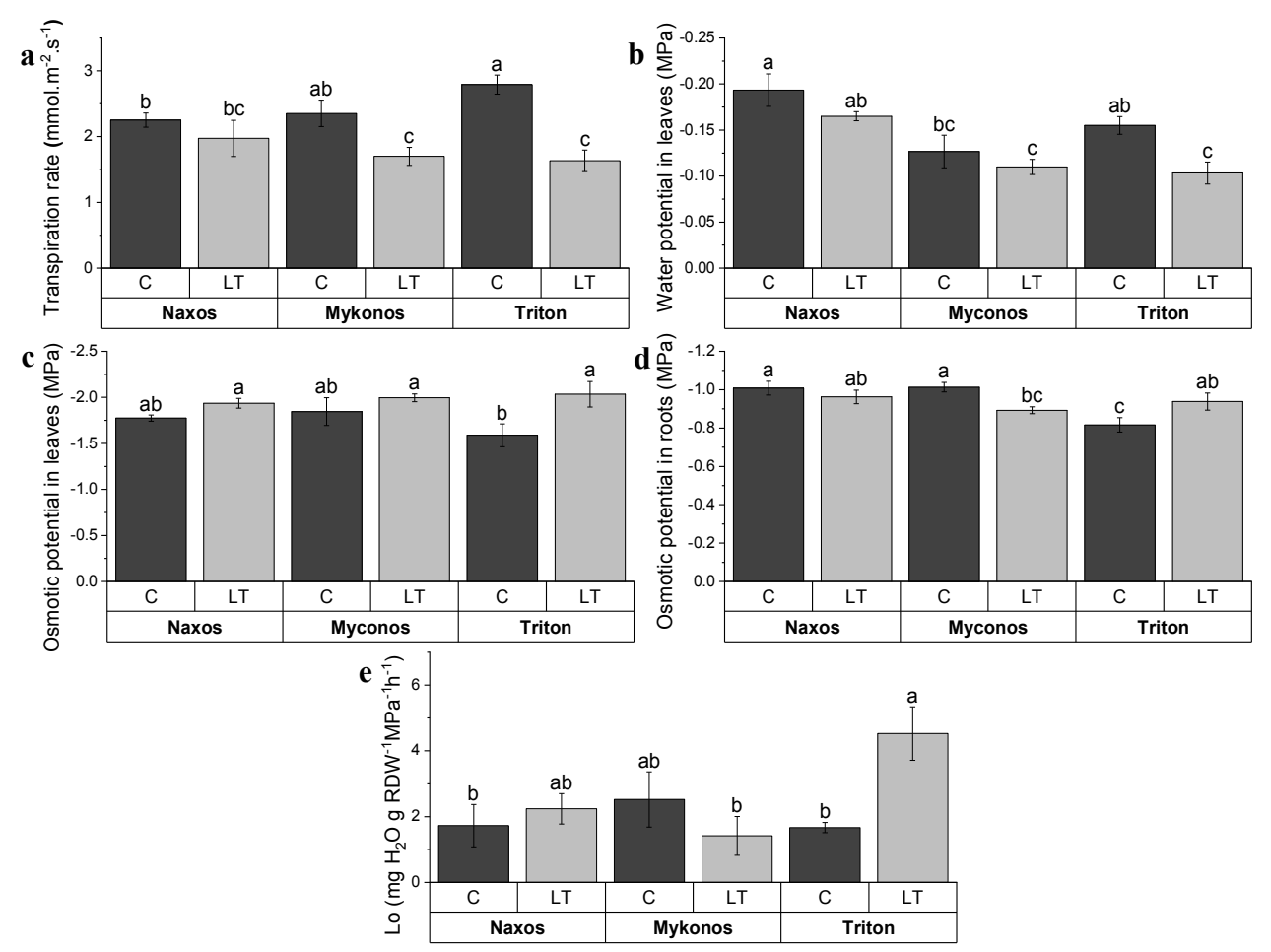

Fig. 2. Transpiration rate (a), water potential in leaves (b), osmotic potential in leaves (c), osmotic potential in roots (d) and root hydraulic conductivity of roots (e) in control conditions (C) and low temperature conditions (LT) for three varieties (Naxos, Mykonos and Triton). Each bar represents the mean of 5 biological replicates \pm SEM. Columns with different letters differ significantly according to Duncan's test $(P=0.05)$. 

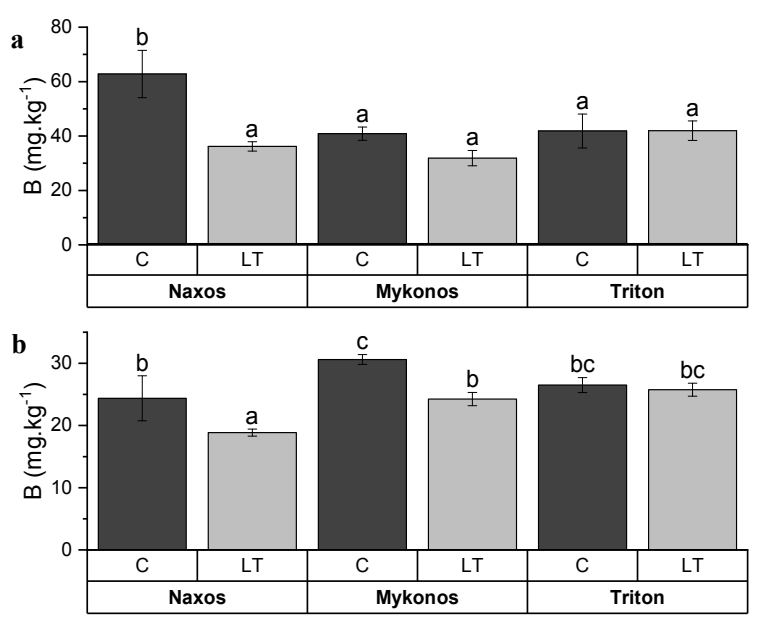

Fig. 3. Boron concentration in leaves (a) and roots (b) for three varieties (Naxos, Mykonos and Triton) in control conditions (C) and low temperature conditions (LT). Each bar represents the mean of 5 biological replicates \pm SEM. Columns with different letters differ significantly according to Duncan's test $(\mathrm{P}=0.05)$.

the other two varieties. For NIP5 (Fig. 4e), the only one that showed significant differences was again Naxos, with a significant increase under LT conditions. For NIP6 (Fig. 4f), two varieties (Mykonos and Triton) increased the expression of this aquaporin in LT respect C, although this was not the case in Naxos.

In roots (Fig. 5) similar results to those found in leaves in relation to PIPs, were found. Both PIP1 (Fig. 5a) and PIP2 (Fig. 5b), had an increased expression in the LT treatment in the Naxos cultivar. TIP1 (Fig. 5c) had a significantly increased expression in the LT treatment in Triton. For TIP2 (Fig. 5d), there was a strong decrease in expression with the LT treatment in the Triton cultivar. NIP5 (Fig. 5e) only showed a significant increase in the Naxos cultivar, as with the expression in the leaves. For NIP6 (Fig. 5f), the three varieties increased the expression of this aquaporin, with the increase in Naxos and Triton being significant.

\section{Discussion}

Studies on cold stress are important, as the cold conditions usually deviate negatively and substantially from the optimal growth temperatures, producing reductions in growth and yield (Krner, 2016). Therefore, the total biomass production of plants serves as an comprehensive index of plant development under adverse conditions (Krner, 2016; Poorter et al., 2009). As expected, the cold treatment reduced the growth of the three cultivars studied. The Mykonos cultivar is commonly cultivated in periods of milder temperatures, and therefore it had higher yields at the optimum temperatures (control conditions), but a lower tolerance to night-time cold conditions (LT treatment). Therefore, the effect of cold stress on fresh weight was much greater in Mykonos than in Naxos or Triton.

The stress imposed in our study diminished the transpiration rate and water potential in the leaves of all the varieties tested, although the decrease was not significant in the Naxos cultivar. In fact, the Naxos cultivar was not significantly affected in any of its physiological parameters. However, the expression of the PIPs (PIP1 and PIP2) considerably increased in both roots and leaves of Naxos plants. In numerous studies, the decrease in PIPs expression has been associated with a reduction in hydraulic conductivity of the root $\left(\mathrm{Lp}_{\mathrm{p}}\right.$ ) (Chaumont et al., 2001; Javot et al., 2003; Martre et al., 2002; Siefritz et al., 2002); PIPs have been shown to improve cold resistance through their overexpression (Sreedharan et al., 2013, Xu et al., 2020), and have also been shown to be involved in cold adaptation when expressed in other hosts (Barzana et al., 2020). Also, the TIP2 in leaves and the NIP5 in roots and leaves experienced an important increase in Naxos plants. The water
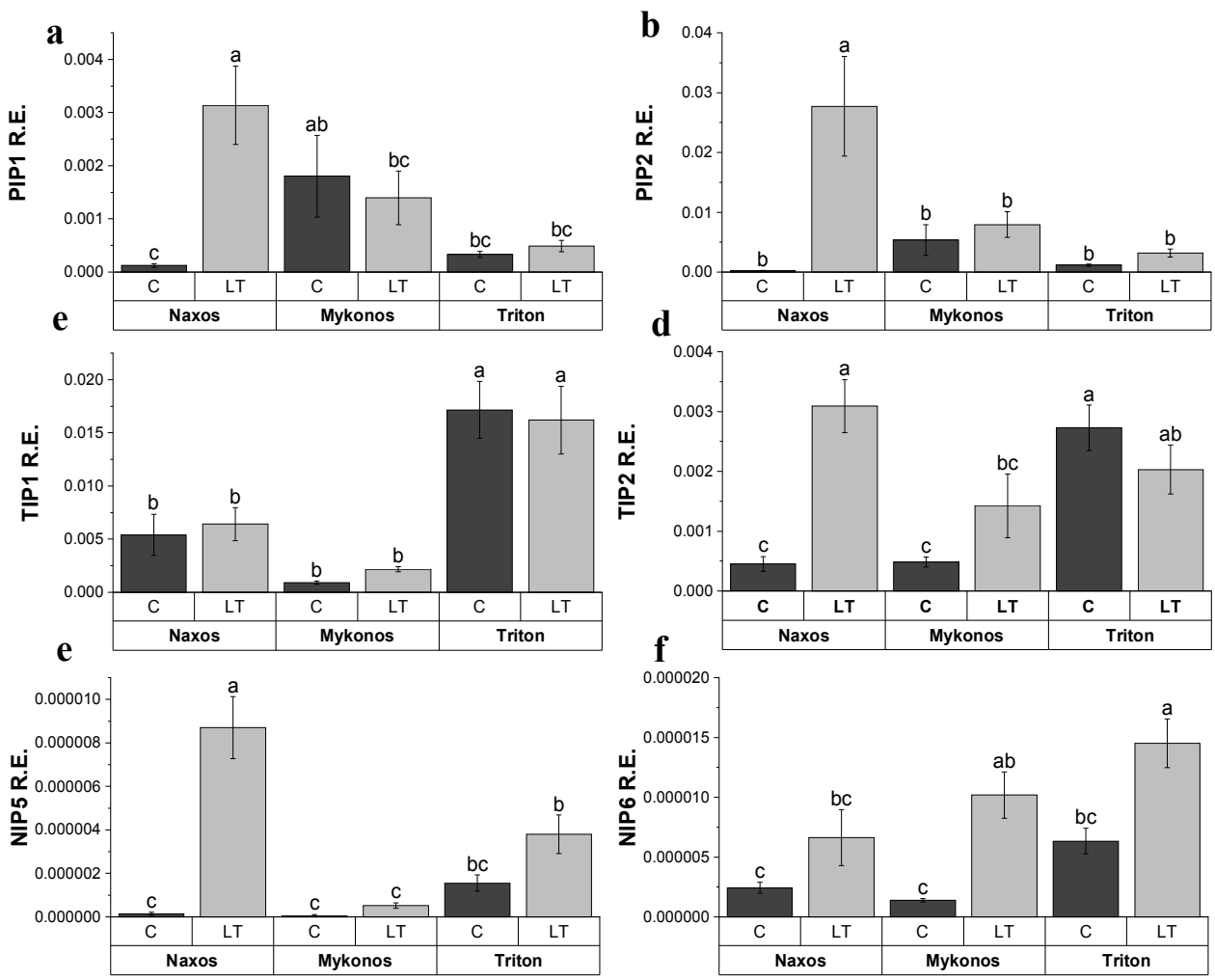

Fig. 4. Aquaporins expression of PIP1 (a), PIP2 (b), TIP1 (c), TIP2 (d), NIP5 (e) and NIP6 (f) in leaves of the three varieties (Naxos, Mykonos and Triton) in control conditions (C) and low temperature conditions (LT). Each bar represents the mean of 4 biological replicates \pm SEM. Columns with different letters differ significantly according to Duncan's test $(\mathrm{P}=0.05)$. 
$\mathbf{a}$
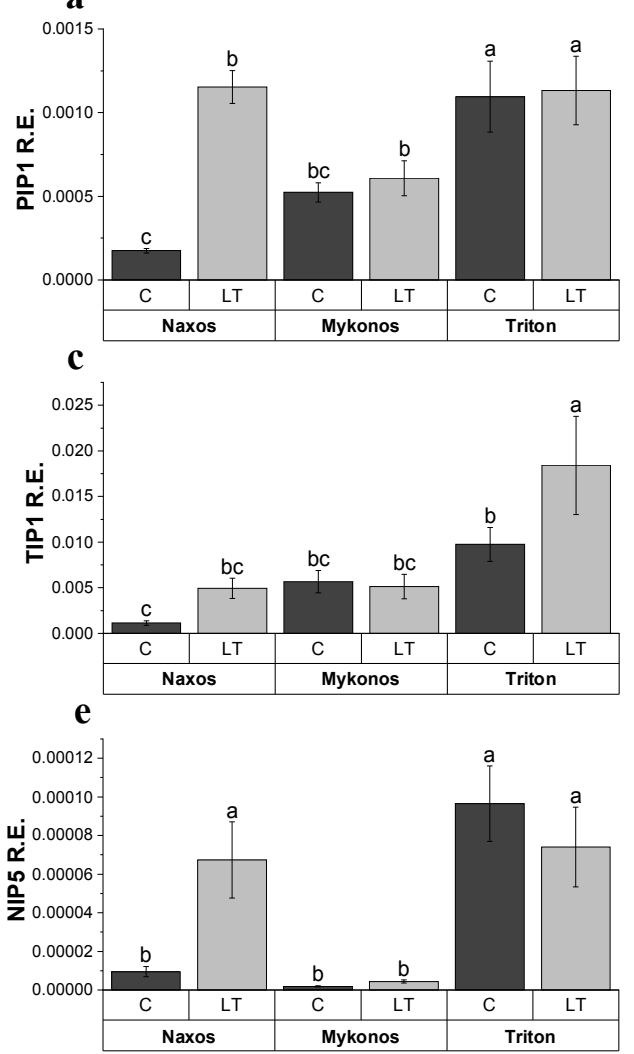

b

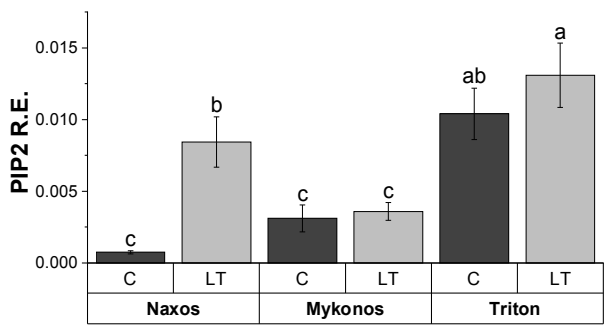

d
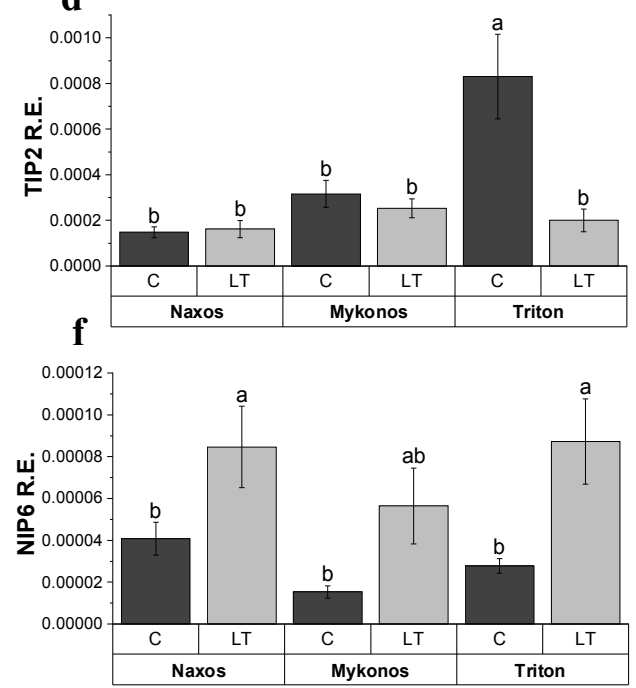

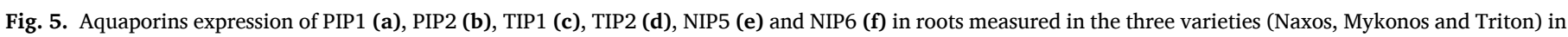

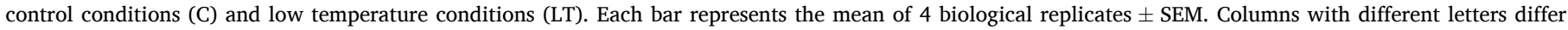
significantly according to Duncan's test $(\mathrm{P}=0.05)$.

transport capacity is maintained in TIPs isoforms, and TIPs2 has been closely related to ammonia $\left(\mathrm{NH}_{3}\right)$ transport. Also, this protein has been suggested to play a role in the detoxification of $\mathrm{N}$ excess into the vacuole and remobilization when it is needed for physiological processes (Bárzana et al., 2014). Likewise, NIP5 has been associated to the uptake of different nutrients by roots, including B (Lopez-Zaplana et al., 2020; Mishra et al., 2018). The increase in the transport through the aquaporins seems to be the reason behind the maintenance of physiological parameters at high levels. Naxos plants also maintained the RWC high under LT conditions, which is a widely used measurement to estimate the hydration status of plants, as it remains high, along with the transpiration and hydraulic conductance processes, to achieve adequate cellular turgor (Joshi et al., 2018; Nayyar and Gupta, 2006). Therefore, it could be proposed that the mechanism adopted by the Naxos plants subjected to LT was a "wasteful strategy", maximizing absorption to take advantage of moments of good water availability (Vilagrosa et al., 2003), by increasing the expression of PIPs, TIPs and NIPs isoforms, allowing it to maintain its physiological functions and tissues hydrated. Undoubtedly, this strategy has a high cost which does not prevent a decrease in its growth under LT conditions.

The Mykonos cultivar grew better under control conditions, as revealed by its total dry and fresh weight. This could be promoted by the highest $\mathrm{L}_{0}$ values observed under control conditions, accompanied by a slightly higher osmotic potential in leaves as reviewed by Martínez-Ballesta et al. (2011). This could be associated to the higher concentrations of PIPs (specially PIP1) under control conditions, as reported for the Parthenon cultivar (Muries et al., 2013). Furthermore, it was the cultivar that was mostly affected by LT conditions, as shown by the highest reduction in weight and a lower water status (measured as relative water content). Mykonos plants subjected to cold stress reduced the transpiration rate and the osmotic potential in the roots, related to the lower root hydraulic conductivity (Aroca et al., 2005; Hussain et al., 2018). In this way, both transport routes (apoplastic and cell-to-cell pathways) were negatively affected, and consequently, the relative water content also decreased. Unlike Naxos, these plants did not show an adjustment through the modulation of the aquaporins PIPs or TIPs, which did not vary, and this lack of adjustment may be responsible for the decreased adaptability to cold stress.

Triton was clearly the slowest growing cultivar under optimal conditions. However, it was the least affected by the LT treatment, thereby showing a great capacity to cope with cold stress. In this cultivar, the transpiration rate significantly diminished by the LT conditions, decreasing the water transport via the apoplastic pathway, as well as the water potential in leaves. However, the decrease in the apoplastic pathway did not lead to a decreased water status or reduced metabolism, as relative water content and fresh weight were not significantly affected. This could be due to a compensation provided by an increased osmotic potential both in roots and leaves, accompanied by the maintenance of PIPs at high levels, in addition to a clear increase in TIP1 expression in root tissues, with the latter being mostly responsible for the permeability of the tonoplast (Morillon and Lassalles, 1999). This compensation seems to give rise to the increased hydraulic conductivity of the roots. It should be noted that Triton had the maximum constitutive expression values for almost all the aquaporins in roots, and the highest constitutive expression of TIPs also in leaf tissues under control conditions. Therefore, it could be proposed that a higher content of aquaporins when the plants are grown in optimal conditions can in turn be an advantage when environmental conditions change, as the plants are intrinsically prepared to react quickly by modifying the activity of aquaporins. This idea was proposed in reverse under drought conditions, where the intrinsic reduced PIPs in optimal conditions avoided water losses when the plants must cope with drought (Bárzana et al., 2015). It 
could therefore be that the intrinsic presence of aquaporins in the membranes are a key adaptation to cope with the natural stress in which the different plants have evolved. Under LT conditions, a strong reduction in the expression of TIP2 was also observed in roots, which could be understood as a mechanism for the retention of nutrients, especially $\mathrm{N}$, in the vacuole. Also, NIP5 was maintained at high levels of expression and a strong increase in the expression of NIP6 was observed. These results could point to an increase in the control of the internal water and nutrients content of the plant through the cell-to-cell transport pathway (Rodríguez-Gamir et al., 2019), thanks to the control of aquaporins that are directly related to the accumulation of water and cellular nutrients. Therefore, Triton plants in LT conditions, although it slows down its growth, continues its development through a "saving strategy" based on minimizing losses (reduced transpiration rate) and activating the accumulation of water and nutrients.

Recently, Porcel et al. (2018) found an aquaporin (BvCOLD1) in Beta vulgaris $\mathrm{L}$. (sugar beet) that confers cold tolerance, relating the expression of boron-transporting aquaporins and resistance to cold. It is known that cold temperatures in the root zone decrease B uptake capacity and B distribution efficiency to the shoot, thereby affecting the normal development of the entire (Huang et al., 2005). Some interactions between $\mathrm{B}$ and low temperatures studied suggest that chilling-induced reduction in plasmalemma hydraulic conductivity, membrane fluidity, activity of aquaporins, and root pressure, contribute to the decrease in root hydraulic conductance, thereby reducing B uptake (Aroca et al., 2001; Asad et al., 2001; Miura and Furumoto, 2013). On the other hand, low temperatures have been shown to induce stomatal dysfunction, negatively affecting B transport from root to shoot. This lack of transport is detrimental for the plant, as B is an essential element for its proper development, as it is part of the cell walls. Also, a lack of B can affect the proper functioning of the plant, as it is also part of the thylakoid membranes, which are essential for proper photosynthesis to occur (Camacho-Cristóbal et al., 2008; El-Shintinawy, 2000; Tavallali, 2017).

In this sense, we studied two aquaporins, NIP5 and NIP6, which are capable of transporting boron, with NIP5;1 being a boric acid channel that is essential for taking up B from the soil (Mishra et al., 2018), and NIP6;1 a protein which facilitates B transport thought the phloem region of vascular tissues (Tanaka et al., 2008). In our study, the plant cultivars that significantly increased the expression of NIP6 kept their B levels stable in leaves (Mykonos and Triton), being Naxos the one that did not significantly modify the expression of NIP6 in leaves which showed a significant decrease in B in the shoot, as reported by Tanaka et al. (2008). However, a relation was not observed between the boron levels and NIP5 expression in the roots, beyond its possible role in maintaining B content in Triton roots at similar levels as the control plants. Once again, the constitutive high expression of NIP5 in roots and leaves and NIP6 in Triton leaf tissues could contribute to the cold tolerance of this cultivar. Nonetheless, NIP5 seems to play a different role aside from B transport. Indeed, some studies have suggested a possible function of NIP5 in the transport of other compounds such as arsenic, antimony, urea, or glycerol, highlighting the versatility of this subgroup of aquaporins, which show great variations in their selectivity depending on the plant studied (Lopez-Zaplana et al., 2020) and thus, no other functions for NIP5 in broccoli should be excluded.

It is important to note that some of the physiological results could be, not directly related to the aquaporins expression, but caused by the functionality of aquaporins, since aquaporins are transmembrane proteins (Heymann and Engel, 2000) and interact closely with the plasma membrane components (Martínez-Ballesta and Carvajal, 2016). In particular, it is known that low temperature affected the physical properties of membranes (Minami et al., 2009) and permeability through the membrane (Chu, 2015). Furthermore, it is known that aquaporins are usually associated with specific domains of membranes, the detergent-resistant plasma membrane fraction (Minami et al., 2009; Yepes-Molina et al., 2020), and changes in the lipid composition of these domains or stress signalling for low temperatures could be altering the positioning of aquaporins, internalizing them and therefore decreasing their functionality (Bellati et al., 2016; Wudick et al., 2015).

In future studies, it would be interesting to perform and in-depth sequencing and characterization of all the aquaporins in broccoli, differentiating between the different isoforms within each subgroup, as not all the aquaporins act the same under cold stress, and some isoforms could perform specific functions in the different strategies adopted by the three varieties against stress.

\section{Conclusions}

In conclusion, the fine control of aquaporins in broccoli has been revealed as essential for responding to low temperatures. Each of the broccoli cultivars utilized different strategies to deal with low temperatures, with Mykonos being the most sensitive among them, and the one with a decreased ability to modulate the expression of aquaporins. Naxos and Triton showed different patterns of behaviour. Naxos plants tried to reverse the stress experienced by increasing the flow of water and nutrients in the periods of optimal conditions, through the increase in aquaporin expression (PIP1, PIP2, NIP5 and NIP6 in roots, and PIP1, PIP2, TIP2 and NIP5 in leaves) following a strategy that we could call "wasteful strategy", with a notably cost in its growth. And Triton showed a "savings strategy" based on decreasing losses by reducing transpiration and controlling water and nutrient transport by the roots. The constitutive high expression of aquaporins seems to be crucial for the cold resistance of the Triton cultivar. Finally, NIP6 appeared to be important aquaporin involved in cold adaptation, both in leaves and roots, mainly due to the increased expression after the LT treatments regardless of the cultivar analysed.

\section{Author's contribution}

Funding was obtained thanks to Micaela Carvajal. Paper conceptualization by Gloria Bárzana. All authors contributed to the study design. The preparation of material, the handling of the samples and measurements, the collection, analysis and interpretation of data were performed by Alvaro Lopez-Zaplana under the direct and participative supervision of Gloria Bárzana. Primers design by Gloria Bárzana. Juan Nicolas-Espinosa prepared all figures and tables and contributed to statistical expertise. All authors contributed to drafting the manuscript and critically revised for important intellectual content. Adjustments, corrections and support from Micaela Carvajal, group director. All authors gave final approval of the article.

\section{Funding}

This work was supported by the Spanish Ministry of Science, Innovation, and Universities (RTC-2017-6119-2), developed under the auspices of the Spanish Higher Council for Scientific Research (CSIC).

\section{CRediT authorship contribution statement}

Alvaro Lopez-Zaplana: Investigation, Writing - original draft, Writing - review \& editing, Visualization. Juan Nicolas-Espinosa: Formal analysis, Data curation, Writing - review \& editing, Visualization. Micaela Carvajal: Resources, Writing - review \& editing, Visualization, Supervision, Project administration, Funding acquisition. Gloria Bárzana: Conceptualization, Methodology, Formal analysis, Investigation, Writing - review \& editing, Visualization.

\section{Declaration of Competing Interest}

The authors declare that they have no known competing financial interests or personal relationships that could have appeared to influence the work reported in this paper. 


\section{Acknowledgements}

The authors thank Mr. Mario Fon for the correction of the English in the manuscript, and SAKATA SEED IBERICA, S.L.U., for providing the seeds.

\section{References}

Ahamed, A., Murai-Hatano, M., Ishikawa-Sakurai, J., Hayashi, H., Kawamura, Y, Uemura, M., 2012. Cold stress-induced acclimation in rice is mediated by rootspecific aquaporins. Plant Cell Physiol. 53, 1445-1456. https://doi.org/10.1093/ pcp/pcs089.

Aroca, R., Tognoni, F., Irigoyen, J.J., Sánchez-Díaz, M., Pardossi, A., 2001. Different roo low temperature response of two maize genotypes differing in chilling sensitivity. Plant Physiol. Biochem. 39, 1067-1073. https://doi.org/10.1016/S0981-9428(01) 01335-3.

Aroca, R., Amodeo, G., Fernández-Illescas, S., Herman, E.M., Chaumont, F., Chrispeels, M.J., 2005. The role of aquaporins and membrane damage in chilling and hydrogen peroxide induced changes in the hydraulic conductance of maize roots. Plant Physiol. 137, 341-353. https://doi.org/10.1104/pp.104.051045.

Aroca, R., Porcel, R., Ruiz-Lozano, J.M., 2007. How does arbuscular mycorrhizal symbiosis regulate root hydraulic properties and plasma membrane aquaporins in Phaseolus vulgaris under drought, cold or salinity stresses? New Phytol. 173, 808-816. https://doi.org/10.1111/j.1469-8137.2006.01961.x.

Asad, A., Bell, R.W., Dell, B., 2001. A critical comparison of the external and internal boron requirements for contrasting species in boron-buffered solution culture. Plant Soil 233, 31-45. https://doi.org/10.1023/A:1010372430137.

Bárzana, G., Aroca, R., Bienert, G.P., Chaumont, F., Ruiz-Lozano, J.M., 2014. New insights into the regulation of aquaporins by the arbuscular mycorrhizal symbiosis in maize plants under drought stress and possible implications for plant performance. Mol. Plant Microbe Interact. 27, 349-363. https://doi.org/10.1094/MPMI-09-130268-R.

Bárzana, G., Aroca, R., Ruiz-Lozano, J.M., 2015. Localized and non-localized effects of arbuscular mycorrhizal symbiosis on accumulation of osmolytes and aquaporins and on antioxidant systems in maize plants subjected to total or partial root drying. Plant Cell Environ. 38, 1613-1627. https://doi.org/10.1111/pce.12507.

Barzana, G., Rios, J.J., Lopez-Zaplana, A., Nicolas-Espinosa, J., Yepes-Molina, L., GarciaIbañez, P., Carvajal, M., 2020. Interrelations of nutrient and water transporters in plants under abiotic stress. Physiol. Plant. https://doi.org/10.1111/ppl.13206 ppl.13206.

Bellati, J., Champeyroux, C., Hem, S., Rofidal, V., Krouk, G., Maurel, C., Santoni, V., 2016. Novel aquaporin regulatory mechanisms revealed by interactomics. Mol. Cell Proteomics 15, 3473-3487. https://doi.org/10.1074/mcp.M116.060087.

Bienert, G.P., Schüssler, M.D., Jahn, T.P., 2008. Metalloids: essential, beneficial or toxic? Major intrinsic proteins sort it out. Trends Biochem. Sci. 33, 20-26. https://doi.org/ 10.1016/j.tibs.2007.10.004

Brulle, F., Bernard, F., Vandenbulcke, F., Cuny, D., Dumez, S., 2014. Identification of suitable qPCR reference genes in leaves of Brassica oleracea under abiotic stresses. Ecotoxicology 23, 459-471. https://doi.org/10.1007/s10646-014-1209-7.

Bussler, W., 2007. Mineral nutrition of plants: principles and perspectives. Zeitschrift für Pflanzenernährung und Bodenkd 132, 158-159. https://doi.org/10.1002/ jpln.19721320211.

Camacho-Cristóbal, J.J., Rexach, J., González-Fontes, A., 2008. Boron in plants: deficiency and toxicity. J. Integr. Plant Biol. 50, 1247-1255. https://doi.org/ 10.1111/j.1744-7909.2008.00742.x.

Chaumont, F., Barrieu, F., Wojcik, E., Chrispeels, M.J., Jung, R., 2001. Aquaporins constitute a large and highly divergent protein family in maize. Plant Physiol. 125 1206-1215. https://doi.org/10.1104/pp.125.3.1206.

Chu, R.K.H., 2015. Temperature-tuned transport in biomembrane pores. J. Mol. Liq. 208, 356-359. https://doi.org/10.1016/j.molliq.2015.05.017.

Dezhabad, F., Haghighi, M., 2020. Bottom-cold stress was less harmful than cold-air stress on tomato seedling production treated with boric acid. Acta Physiol. Plant. 42, 1-13. https://doi.org/10.1007/s11738-020-3035-2.

Diehn, T.A., Pommerrenig, B., Bernhardt, N., Hartmann, A., Bienert, G.P., 2015. Genomewide identification of aquaporin encoding genes in Brassica oleracea and their phylogenetic sequence comparison to Brassica crops and Arabidopsis. Front. Plant Sci. 6, 166. https://doi.org/10.3389/fpls.2015.00166.

Ding, L., Milhiet, T., Couvreur, V., Nelissen, H., Meziane, A., Parent, B., Aesaert, S., Van Lijsebettens, M., Inzé, D., Tardieu, F., Draye, X., Chaumont, F., 2020. Modification of the expression of the aquaporin ZmPIP2;5 affects water relations and plant growth Plant Physiol. 182, 2154-2165. https://doi.org/10.1104/PP.19.01183.

El-Shintinawy, F., 2000. Structural and functional damage caused by boron deficiency in sunflower leaves. Photosynthetica 36, 565-573. https://doi.org/10.1023/A: 1007096105491.

Fowler, S., Thomashow, M.F., 2002. Arabidopsis transcriptome profiling indicates that multiple regulatory pathways are activated during cold acclimation in addition to the CBF cold response pathway. Plant Cell 14, 1675-1690. https://doi.org/10.1105/ tpc.003483.

Francisco, M., Tortosa, M., Martínez-Ballesta, M.C., Velasco, P., García-Viguera, C., Moreno, D.A., 2017. Nutritional and phytochemical value of Brassica crops from the agri-food perspective. Ann. Appl. Biol. 170, 273-285. https://doi.org/10.1111/ aab.12318.

Heymann, J.B., Engel, A., 2000. Structural clues in the sequences of the aquaporins. J. Mol. Biol. 295, 1039-1053. https://doi.org/10.1006/jmbi.1999.3413.
Huang, L., Ye, Z., Bell, R.W., Dell, B., 2005. Boron nutrition and chilling tolerance of warm climate crop species. Ann. Bot. 96, 755-767. https://doi.org/10.1093/aob/ mci228.

Huang, C., Zhou, S., Hu, W., Deng, X., Wei, S., Yang, G., He, G., 2014. The wheat aquaporin gene TaAOP7 confers tolerance to cold stress in transgenic tobacco. Zeitschrift fur Naturforsch. - Sect. C J. Biosci. 69, 142-148. https://doi.org/10.5560/ ZNC.2013-0079.

Hussain, H.A., Hussain, S., Khaliq, A., Ashraf, U., Anjum, S.A., Men, S., Wang, L., 2018. Chilling and drought stresses in crop plants: implications, cross talk, and potential management opportunities. Front. Plant Sci. 9, 393. https://doi.org/10.3389/ fpls.2018.00393.

Jang, J.Y., Kim, D.G., Kim, Y.O., Kim, J.S., Kang, H., 2004. An expression analysis of a gene family encoding plasma membrane aquaporins in response to abiotic stresses in Arabidopsis thaliana. Plant Mol. Biol. 54, 713-725. https://doi.org/10.1023/B: PLAN.0000040900.61345.a6.

Javot, H., Lauvergeat, V., Santoni, V., Martin-Laurent, F., Güçlü, J., Vinh, J., Heyes, J., Franck, K.I., Schäffner, A.R., Bouchez, D., Maurel, C., 2003. Role of a single aquaporin isoform in root water uptake. Plant Cell 15, 509-522. https://doi.org/ $10.1105 /$ tpc.008888.

Jeon, J., Kim, J., 2013. Cold stress signaling networks in Arabidopsis. J. Plant Biol. 56, 69-76. https://doi.org/10.1007/s12374-013-0903-y.

Joshi, A., Kaur, S., Dharamvir, K., Nayyar, H., Verma, G., 2018. Multi-walled carbon nanotubes applied through seed-priming influence early germination, root hair, growth and yield of bread wheat (Triticum aestivum L.). J. Sci. Food Agric. 98, 3148-3160. https://doi.org/10.1002/jsfa.8818.

Köse, C., Günes, A., Kaya, Ö., Kıtır, N., Turan, M., Sahin, F., 2018. Freeze injure and antioxidant enzyme activity of grapevine (Vitis Vinifera) under bio-boron fertilizer applications. Erwerbs-Obstbau 60, 3-10. https://doi.org/10.1007/s10341-0180370-1.

Krner, C., 2016. Plant adaptation to cold climates. F1000Research, p. 5. https://doi.org/ 10.12688/f1000research.9107.1.

Kumar, K., Pilani, B., Goa, B., Mosa, K.A., Parkash Dhankher, O., 2014. Two rice plasma membrane intrinsic proteins, OsPIP2;4 and OsPIP2;7, are involved in transport and providing tolerance to boron toxicity. Planta 239, 187-198. https://doi.org/ 10.1007/s00425-013-1969-y.

Livak, K.J., Schmittgen, T.D., 2001. Analysis of relative gene expression data using realtime quantitative PCR and the 2- $\Delta \Delta \mathrm{CT}$ method. Methods 25, 402-408. https://doi. org/10.1006/meth.2001.1262.

Lopez-Zaplana, A., Nicolas-Espinosa, J., Carvajal, M., Bárzana, G., 2020. Genome-wide analysis of the aquaporin genes in melon (Cucumis melo L.). Sci. Rep. 10, 22240. https://doi.org/10.1038/s41598-020-79250-w.

Martínez-Ballesta, M.C., Carvajal, M., 2016. Mutual interactions between aquaporins and membrane components. Front. Plant Sci. 7, 1322. https://doi.org/10.3389/ fpls. 2016.01322.

Martínez-Ballesta, M.C., Rodríguez-Hernández, M.C., Alcaraz-López, C., MotaCadenas, C., Muries, B., Carvajal, M., 2011. Plant hydraulic conductivity: the aquaporins contribution. In: Elango, L. (Ed.), Hydraulic Conductivity: Issues, Determination and Applications. InTech, Rijeka, Croatia, pp. 103-122.

Martre, P., Morillon, R., Barrieu, F., North, G.B., Nobel, P.S., Chrispeels, M.J., 2002. Plasma membrane aquaporins play a significant role during recovery from water deficit 1. Am Soc Plant Biol 130, 2101-2110. https://doi.org/10.1104/pp.009019.

Maurel, C., Verdoucq, L., Luu, D.-T., Santoni, V., 2008. Plant aquaporins: membrane channels with multiple integrated functions. Annu. Rev. Plant Biol. 59, 595-624. https://doi.org/10.1146/annurev.arplant.59.032607.092734.

Maurel, C., Boursiac, Y., Luu, D.T., Santoni, V., Shahzad, Z., Verdoucq, L., 2015. Aquaporins in plants. Physiol. Rev. 95, 1321-1358. https://doi.org/10.1152/ physrev.00008.2015.

Minami, A., Fujiwara, M., Furuto, A., Fukao, Y., Yamashita, T., Kamo, M., Kawamura, Y., Uemura, M., 2009. Alterations in detergent-resistant plasma membrane microdomains in arabidopsis thaliana during cold acclimation. Plant Cell Physiol. 50, 341-359. https://doi.org/10.1093/pcp/pcn202.

Mishra, S., Heckathorn, S.A., Frantz, J.M., Krause, C., 2018. The effect of boron availability, $\mathrm{CO} 2$, and irradiance on relative accumulation of the major boron transport proteins, BOR1 and NIP5;1. Biol. Plant. 62, 121-128. https://doi.org/ 10.1007/s10535-017-0744-5.

Miura, K., Furumoto, T., 2013. Cold signaling and cold response in plants. Int. J. Mol. Sci. 14, 5312-5337. https://doi.org/10.3390/ijms14035312.

Moreno, D.A., Carvajal, M., López-Berenguer, C., García-Viguera, C., 2006. Chemical and biological characterisation of nutraceutical compounds of broccoli. J. Pharm. Biomed. Anal. 41, 1508-1522. https://doi.org/10.1016/j.jpba.2006.04.003.

Morillon, R., Lassalles, J.P., 1999. Osmotic water permeability of isolated vacuoles. Planta 210, 80-84. https://doi.org/10.1007/s004250050656.

Mosa, K., Kumar, K., Chhikara, S., Musante, C., White, J., Chankher, O., 2016. Enhanced boron tolerance in plants mediated by bidirectional transport through plasma membrane intrinsic proteins. Sci. Rep. 6, 21640. https://doi.org/10.1038/ srep 21640.

Murai-Hatano, M., Kuwagata, T., Sakurai, J., Nonami, H., Ahamed, A., Nagasuga, K., Matsunami, T., Fukushi, K., Maeshima, M., Okada, M., 2008. Effect of low root temperature on hydraulic conductivity of rice plants and the possible role of aquaporins. Plant Cell Physiol. 49, 1294-1305. https://doi.org/10.1093/pcp/ pcn104.

Muries, B., Faize, M., Carvajal, M., Martínez-Ballesta, M.C., 2011. Identification and differential induction of the expression of aquaporins by salinity in broccoli plants. Mol. Biosyst. 7, 1322-1335. https://doi.org/10.1039/c0mb00285b. 
Muries, B., Carvajal, M., Martínez-Ballesta, M.C., 2013. Response of three broccoli cultivars to salt stress, in relation to water status and expression of two leaf aquaporins. Planta 237, 1297-1310. https://doi.org/10.1007/s00425-013-1849-5.

Nasehzadeh, M., Ellis, R.H., 2017. Wheat seed weight and quality differ temporally in sensitivity to warm or cool conditions during seed development and maturation. Ann. Bot. 120, 479-493. https://doi.org/10.1093/aob/mcx074.

Nayyar, H., Gupta, D., 2006. Differential sensitivity of C3 and C4 plants to water deficit stress: association with oxidative stress and antioxidants. Environ. Exp. Bot. 58, 106-113. https://doi.org/10.1016/j.envexpbot.2005.06.021.

Osakabe, Y., Osakabe, K., Shinozaki, K., Tran, L.S.P., 2014. Response of plants to water stress. Front. Plant Sci. 5, 86. https://doi.org/10.3389/fpls.2014.00086.

Poorter, H., Niinemets, Ü., Poorter, L., Wright, I.J., Villar, R., 2009. Causes and consequences of variation in leaf mass per area (LMA): a meta-analysis. New Phytol. 182, 565-588. https://doi.org/10.1111/j.1469-8137.2009.02830.x.

Porcel, R., Bustamante, A., Ros, R., Serrano, R., Mulet Salort, J.M., 2018. BvCOLD1: A novel aquaporin from sugar beet (Beta vulgaris L.) involved in boron homeostasis and abiotic stress. Plant Cell Environ. 41, 2844-2857. https://doi.org/10.1111/ pce.13416.

Ramakers, C., Ruijter, J.M., Lekanne Deprez, R.H., Moorman, A.F.M., 2003. Assumptionfree analysis of quantitative real-time polymerase chain reaction (PCR) data. Neurosci. Lett. 339, 62-66. https://doi.org/10.1016/S0304-3940(02)01423-4.

Rodríguez-Gamir, J., Xue, J., Clearwater, M.J., Meason, D.F., Clinton, P.W., Domec, J.-C. 2019. Aquaporin regulation in roots controls plant hydraulic conductance, stomatal conductance, and leaf water potential in Pinus radiata under water stress. Plant Cell Environ. 42, 717-729. https://doi.org/10.1111/pce.13460.

Sanghera, G.S., Wani, S.H., Hussain, W., Singh, N.B., 2011. Engineering cold stress tolerance in crop plants. Curr. Genomics 12, 30. https://doi.org/10.2174/ 138920211794520178.

Siefritz, F., Tyree, M.T., Lovisolo, C., Schubert, A., Kaldenhoff, R., 2002. PIP1 plasma membrane aquaporins in tobacco: from cellular effects to function in plants. Plant Cell 14, 869-876. https://doi.org/10.1105/tpc.000901.

Steudle, E., 2001. The cohesion-tension mechanism and the acquisition of water by plant roots. Annu. Rev. Plant Physiol. Plant Mol. Biol. 52, 847-875. https://doi.org/ 10.1146/annurev.arplant.52.1.847.

Takano, J., Wada, M., Ludewig, U., Schaaf, G., Von Wirén, N., Fujiwara, T., 2006. The Arabidopsis major intrinsic protein NIP5; 1 is essential for efficient boron uptake and plant development under boron limitation. Plant Cell 18, 1498-1509. https://doi. org/10.1105/tpc.106.041640.
Tanaka, M., Wallace, I.S., Takano, J., Roberts, D.M., Fujiwara, T., 2008. NIP6;1 is a boric acid channel for preferential transport of boron to growing shoot tissues in Arabidopsis. Plant Cell 20, 2860-2875. https://doi.org/10.1105/tpc.108.058628.

Tavallali, V., 2017. Interactive effects of zinc and boron on growth, photosynthesis, and water relations in pistachio. J. Plant Nutr. 40, 1588-1603. https://doi.org/10.1080/ 01904167.2016.1270308.

Turner, N.C., 1988. Irrigation clence measurement of plant water status by the pressure chamber technique. Irrig. Sci. 9, 289-308.

Tyerman, S.D., Niemietz, C.M., Bramley, H., 2002. Plant aquaporins: Multifunctional water and solute channels with expanding roles. Plant Cell Environ. 25, 173-194. https://doi.org/10.1046/j.0016-8025.2001.00791.x.

Vandesompele, J., De Preter, K., Pattyn, F., Poppe, B., Van Roy, N., De Paepe, A., Speleman, F., 2002. Accurate normalization of real-time quantitative RT-PCR data by geometric averaging of multiple internal control genes. Genome Biol. 3, 1-12. https://doi.org/10.1186/gb-2002-3-7-research0034.

Vilagrosa, A., Bellot, J., Vallejo, V.R., Gil-Pelegrín, E., 2003. Cavitation, stomatal conductance, and leaf dieback in seedlings of two co-occurring Mediterranean shrubs during an intense drought. J. Exp. Bot. 54, 2015-2024. https://doi.org/ 10.1093/jxb/erg221.

Wang, M., Ding, L., Gao, L., Li, Y., Shen, Q., Guo, S., 2016. The interactions of aquaporins and mineral nutrients in higher plants. Int. J. Mol. Sci. 17, 1229. https://doi.org/ 10.3390/ijms17081229.

Wong, C.E., Li, Y., Labbe, A., Guevara, D., Nuin, P., Whitty, B., Diaz, C., Golding, G.B., Gray, G.R., Weretilnyk, E.A., Griffith, M., Moffatt, B.A., 2006. Transcriptional profiling implicates novel interactions between abiotic stress and hormonal responses in Thellungiella, a close relative of Arabidopsis. Plant Physiol. 140, 1437-1450. https://doi.org/10.1104/pp.105.070508.

Wudick, M.M., Li, X., Valentini, V., Geldner, N., Chory, J., Lin, J., Maurel, C., Luu, D.T., 2015. Subcellular redistribution of root aquaporins induced by hydrogen peroxide. Mol. Plant 8, 1103-1114. https://doi.org/10.1016/j.molp.2015.02.017.

Xu, Y., Hu, W., Liu, J., Song, S., Hou, X., Jia, C., Li, J., Miao, H., Wang, Z., Tie, W., Xu, B., Jin, Z., 2020. An aquaporin gene MaPIP2-7 is involved in tolerance to drought, cold and salt stresses in transgenic banana (Musa acuminata L.). Plant Physiol. Biochem. 147, 66-76. https://doi.org/10.1016/j.plaphy.2019.12.011.

Yepes-Molina, L., Carvajal, M., Martínez-Ballesta, M.C., 2020. Detergent resistant membrane domains in broccoli plasma membrane associated to the response to salinity stress. Int. J. Mol. Sci. 21, 7694. https://doi.org/10.3390/ijms21207694. 